\title{
Cyclopamine Bioactivity by Extraction Method from Veratrum californicum
}

Matthew W. Turner, ${ }^{\text {a,b }}$ Roberto Cruz, ${ }^{\mathrm{a}}$ Jared Mattos, ${ }^{\mathrm{a}}$ Nic Baughman, ${ }^{\mathrm{a}}$ Jordan Elwell, ${ }^{\mathrm{a}}$ Jenny Fothergill, ${ }^{a}$ Anna Nielsen, ${ }^{a}$ Jessica Brookhouse, ${ }^{a}$ Ashton Bartlett, ${ }^{a}$ Petr Malek,,${ }^{a}$ Xinzhu Pu ${ }^{\mathrm{c}}$ Matthew D. King, ${ }^{\mathrm{a}}$ and Owen M. McDougal ${ }^{\mathrm{a}, *}$

${ }^{a}$ Department of Chemistry and Biochemistry, Boise State University, 1910 University Drive, Boise, Id 83725

${ }^{b}$ Biomolecular Sciences Ph.D. Program, Boise State University, 1910 University Drive, Boise, Id 83725

'Biomolecular Research Center, Boise State University, 1910 University Drive, Boise, Id 83725

*Corresponding Author: Owen M. McDougal, Email: owenmcdougal@boisestate.edu, Phone: (208) 426-3964

\begin{abstract}
Veratrum californicum, commonly referred to as corn lily or Californian false hellebore, grows in high mountain meadows and produces the steroidal alkaloid cyclopamine, a potent inhibitor of the Hedgehog $(\mathrm{Hh})$ signaling pathway. The Hh pathway is a crucial regulator of many fundamental processes during vertebrate embryonic development. However, constitutive activation of the Hh pathway contributes to the progression of various cancers. In the present study, a direct correlation was made between the extraction efficiency for cyclopamine from root and rhizome by eight methods, and the associated biological activity in Shh-Light II cells using the Dual-Glo® Luciferase Assay System. Alkaloid recovery ranged from 0.39-8.03 mg/g, with ethanol soak being determined to be the superior method for obtaining biologically active
\end{abstract}


cyclopamine. Acidic ethanol and supercritical extractions yielded degraded or contaminated cyclopamine with lower antagonistic activity towards Hh signaling.

\section{Graphical Abstract}

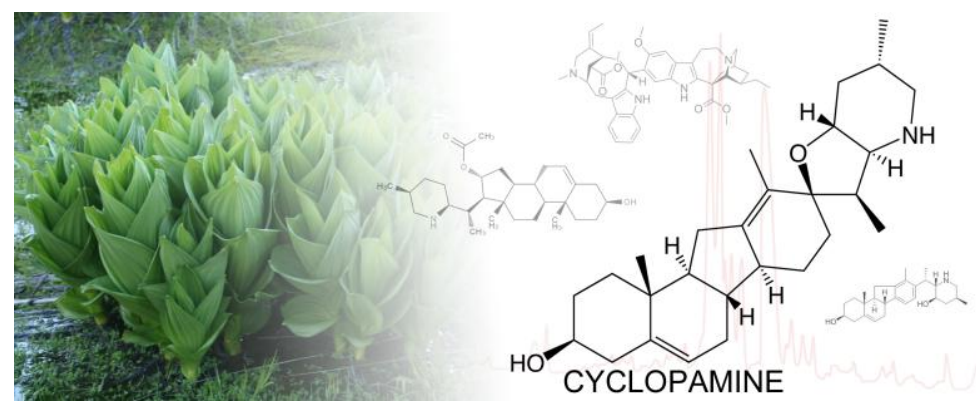

\section{Introduction}

Veratrum californicum (V. californicum), a plant that is rich in steroidal alkaloids, is native to moist, high elevation regions in the western United States. ${ }^{1-2}$ Of these alkaloids, cyclopamine has been studied for its effect as a teratogen ${ }^{3-5}$ through antagonism of the Sonic Hedgehog (Shh) signaling pathway, ${ }^{6-8}$ which is principally active during fetal development. Inhibition of Shh signaling has gained recent interest due to the discovery that aberrant pathway activation is significant in the progression of over twenty cancers ${ }^{2,9,10}$ including prostate, ${ }^{11}$ gallbladder, ${ }^{12}$ pancreatic, ${ }^{13}$ and basal cell carcinoma. ${ }^{14}$ Active drug development in this field has either modeled cancer chemotherapeutics after cyclopamine, as in the case of vismodegib developed by Genentec, ${ }^{15}$ or has synthetically altered the natural product, as was done by Infinity Pharmaceuticals to make IPI-926, ${ }^{16}$ a drug candidate that has undergone phase two clinical trials for treatment of recurrent head and neck cancers. ${ }^{17}$

Continued development of Shh inhibitors may increase demand for harvesting $V$. californicum as a natural source of cyclopamine, primarily from roots and rhizomes. Preliminary 
studies to extract cyclopamine from $V$. californicum were performed by soaking the biomass in benzene for 36 hours. ${ }^{18}$ The extraction efficiency of benzene was improved by switching from soaking the biomass for long periods of time to employing Soxhlet reflux. ${ }^{19}$ Additional efforts to reduce extraction time and improve product yield include microwave assisted extraction, or alternatively, deglycosylation of cycloposine prior to collection of cyclopamine. ${ }^{20-22}$

Chandler et al. ${ }^{22}$ took advantage of the high solubility of alkaloids in ethanol and the improved extraction efficiency using Soxhlet to obtain nearly three-fold increase in the amount of cyclopamine as compared to traditional benzene extraction. While higher yields were achieved by ethanol extraction, it was not determined if the collected cyclopamine retained biological activity. Cyclopamine has been reported to degrade to veratramine or inactive isomers under acidic, aqueous conditions. ${ }^{23-24}$ Literature and patent review provide no comprehensive investigation to correlate extraction efficiency to the potency of Shh inhibition.

In the current study, the total yield and biological activity of cyclopamine recovered from V. californicum root and rhizome using eight extraction techniques is correlated directly to alkaloid activity. Extractions were performed by Soxhlet reflux with benzene, Soxhlet reflux with ethanol in acidic, neutral, and alkaline conditions, ethanol soak, benzene soak, supercritical fluid, and ethanolic microwave assisted extraction (MAE). Cyclopamine was isolated by high pressure liquid chromatography (HPLC), and biological activity was tested using Shh-Light II cells.

\section{Materials and Methods}

\section{Extraction Methods}


Solvents and Reagents: Extraction solvents, $95 \% \mathrm{EtOH}$, benzene, $\mathrm{HCl}$, and $\mathrm{NH}_{4} \mathrm{OH}$ were purchased from Fisher Scientific (Pittsburgh, Pennsylvania). HPLC mobile phase consisted of 18 $\mathrm{M} \Omega \mathrm{H}_{2} \mathrm{O}$ and HPLC grade trifluoroacetic acid (TFA), formic acid and acetonitrile (>99\% purity, Fisher Scientific). Alkaloid standards for cyclopamine and veratramine were purchased from Logan Natural Products (Logan, Utah), and additional cyclopamine was purchased from LC Laboratories (Woburn, Massachusetts) and Alfa Aesar (Ward Hill, Massachusetts).

Obtaining and Preparing Biomass: A complete specimen of V. californicum was harvested at an elevation of $2134 \mathrm{~m}(\sim 7000 \mathrm{ft})$, from a northwest facing slope, growing in a moist meadow near the Elk Meadows trail at Bogus Basin Mountain Resort, located in the Boise National Forest, Idaho. The leaf and stalk of the plant were separated from the rhizome and roots, and all plant parts were cut into smaller pieces to fit into quart size Ziploc bags. The sealed bags were placed in a cooler on a bed of ice for transportation. The biomass was collected at a late stage in the plant's life cycle; the plant had noticeable brown edges along its leaves and top indicating annual deterioration of above ground material in preparation for winter. Within two hours and upon arrival in the lab, the roots and rhizomes were chopped into $2 \mathrm{~cm}$ segments and dried for 14 hours using a LabConco Freezone 4.5 freeze drying unit, followed by storage at $-20{ }^{\circ} \mathrm{C}$. Prior to usage, the frozen biomass was again lyophilized to ensure dryness, flash frozen in liquid nitrogen, and ground to a fine powder by mortar and pestle. The homogenized biomass from the same plant was used to perform each of the following extraction methods in triplicate. Soxhlet Reflux (Ethanol and Benzene): Approximately $2.0 \mathrm{~g}$ of pulverized biomass was packed inside a $25 \mathrm{~mL}$ cellulose thimble, which was placed into a Soxhlet column, and fitted with a 500 $\mathrm{ml}$ round bottom flask containing $150 \mathrm{ml}$ of either ethanol or benzene. The Soxhlet reflux was 
maintained for 6 hours. After six hours, the reflux solvent turns a darker amber color, and becomes a syrup-like consistency following rotary evaporation.

Soxhlet Reflux (Acidic and Basic): Approximately $2.0 \mathrm{~g}$ of pulverized biomass was packed inside a $25 \mathrm{~mL}$ cellulose thimble, which was placed into a Soxhlet column, and fitted with a $500 \mathrm{ml}$ round bottom flask containing $150 \mathrm{ml}$ of either 98.3:1.7 ethanol: ammonium hydroxide or 98.3:1.7 ethanol: formic acid, to achieve extraction conditions at $\mathrm{pH}$ values of 10 and 4, respectively. The Soxhlet reflux was maintained for 6 hours. After six hours, the reflux solvent turns a darker amber color, and becomes a syrup-like consistency following rotary evaporation.

Soak Extraction (Ethanol and Benzene): Approximately $2.0 \mathrm{~g}$ of pulverized biomass was added to a $250 \mathrm{ml}$ round bottom flask followed by $150 \mathrm{~mL}$ of ethanol or benzene. The resultant slurry was sonicated for 30 minutes and then stirred for 24 hours on a stir plate. The amber extract was vacuum filtered through $0.45 \mu \mathrm{m}$ Whatman filter paper.

Supercritical Fluid Extraction: A tight coil of copper wire was placed inside a $15 \mathrm{~mL}$ falcon tube with a handle to help lower the coil. The wire was lowered leaving a $1 \mathrm{~mL}$ gap between the coil and the bottom of the tube. Two grams of biomass were packed firmly on top of the copper coil. Dry ice was ground into a powder and packed to the top of the tube. The cap was firmly sealed and the entire tube was submerged in warm tap water. The reaction was considered complete when solid and liquid $\mathrm{CO}_{2}$ were no longer present and bubbles ceased to appear in the submersion tank. Dry ice was added an additional three times to ensure complete extraction. The biomass and filtrate were rinsed with ethanol to remove residue from the tube. Ethanol fractions were combined for later chemical analysis. 
Microwave Extraction: Microwave assisted extraction method was performed on $2.0 \mathrm{~g}$ pulverized biomass using $50 \mathrm{~mL}$ of $67 \%$ ethanol and $33 \%$ water (v/v). Sodium carbonate (15 $\mathrm{g} / \mathrm{L}$ ) was added to ensure alkaline conditions ( $\mathrm{pH} 10)$. The microwave assisted extraction was performed using a CEM MARS 5 system. The reaction vessel was heated from room temperature to $120{ }^{\circ} \mathrm{C}$ over three minutes. The temperature was held at $120{ }^{\circ} \mathrm{C}$ for three minutes using a power of $100 \mathrm{~W}$. A subsequent 20 minute cool-down period resulted in a solution temperature of less than $55^{\circ} \mathrm{C}$. The solution was transferred to a $250 \mathrm{~mL}$ round bottom flask and evaporated to approximately $20 \%(\sim 10 \mathrm{~mL})$ of its original volume using a rotary evaporator (35$50{ }^{\circ} \mathrm{C}$ ) and then vacuum filtered.

Crude Product Preparation: The crude extract obtained from each method was dissolved in $10 \mathrm{ml}$ of ethanol, and the solution was warmed and sonicated to achieve complete dissolution. Addition of $\mathrm{NH}_{4} \mathrm{OH}$ achieved alkaline solvent conditions of $\mathrm{pH}>10$. The aqueous solution was added directly to a supported liquid extraction (SLE) column (Chem Elut, Agilent, Santa Clara, California) and allowed to adsorb for 10 minutes, followed by elution of alkaloids with chloroform $(3 \times 10 \mathrm{~mL})$ using a vacuum manifold set to a pressure of $2 \mathrm{mbar}$. The chloroform fractions were combined, filtered, and evaporated to dryness. All samples were dissolved in 1 $\mathrm{mL}$ ethanol as a mixture of alkaloids.

\section{Purification, Quantification, and Qualitative Analysis of Cyclopamine from Extracts} Cyclopamine Purification: HPLC was used to purify cyclopamine from the alkaloid mixture using a Dionex UltiMate ${ }^{\circledR} 3000$ uHPLC system coupled to a diode array detector (DAD) and an automated fraction collector. A semi-preparative Agilent Zorbax SB-C ${ }_{18}$ column $(9.4 \times 250 \mathrm{~mm}$, $5 \mu \mathrm{m}$ ) was used to achieve separation. The mobile phase was $0.1 \%$ TFA in water (Buffer A) and acetonitrile (Buffer B) with a flow rate of $3.0 \mathrm{ml} / \mathrm{min}$. The linear gradient method was used to 
separate alkaloids from the mixture starting at 5\% acetonitrile and ending at 90\% acetonitrile over 25 minutes. Cyclopamine isolated by this procedure was stored as a dried solid at $-20{ }^{\circ} \mathrm{C}$ for use in bioactivity studies and mass spectrometry analysis.

Cyclopamine Quantification: Aliquots of crude alkaloid extracts were used to quantitate cyclopamine content using a charge aerosol detector (CAD), and MSQ Plus mass spectrophotometer equipped with a Thermo Acclaim $120 \mathrm{C}_{18}$ column $(2.1 \times 150 \mathrm{~mm}, 3 \mu \mathrm{m})$. Buffer and gradient conditions were the same as stated above, but the flow rate was decreased to $0.3 \mathrm{ml} / \mathrm{min}$. Cyclopamine standard was used to create a calibration curve at concentrations of 0.5 , 1.0, 2.5, 5.0 and $10.0 \mathrm{mM}$ with detection recorded by a Corona Veo RS CAD with the power function set to 1.70 . The quantity of cyclopamine was determined from the alkaloid mixtures obtained from each extraction method performed in triplicate, and the extraction efficiency and the standard deviation was calculated.

Qualitative Analysis of Cyclopamine: Cyclopamine isolated from each alkaloid mixture was analyzed by mass spectrometry using an ultra-high resolution Quadrupole Time of Flight (QTOF) instrument (Bruker maXis). The electrospray ionization (ESI) source was operated under the following conditions: positive ion mode; nebulizer pressure: $0.8 \mathrm{Bar}$; flow rate of drying gas $\left(\mathrm{N}_{2}\right)$ : $4 \mathrm{~L} / \mathrm{min}$; drying gas temperature: $200{ }^{\circ} \mathrm{C}$; voltage between $\mathrm{HV}$ capillary and $\mathrm{HV}$ end-plate offset: $3000 \mathrm{~V}$ to $-500 \mathrm{~V}$; mass range was set from 80 to $1000 \mathrm{~m} / \mathrm{z}$; and the quadrupole ion energy was $4.0 \mathrm{eV}$. Samples were analyzed by direct infusion with a syringe pump at a flow rate of $240 \mu \mathrm{L} / \mathrm{hr}$. Sodium formate was used to calibrate the system in the mass range. Spectra were collected for intact parent ions followed by isolation and fragmentation using collision induced decay MS/MS over a range of collision energies $(0-40 \mathrm{eV})$. Fragmentation patterns were 
compared to cyclopamine and veratramine standards. Data were analyzed using the Compass

Data Analysis software package (Bruker Corporation, Billerica, Massachusetts).

Biological Activity of Purified Extracts

Cell Culture: Shh-Light II cells (JHU-068) were maintained in Dulbecco's Modified Eagle Medium (DMEM) (Gibco) supplemented with $0.4 \mathrm{mg} / \mathrm{mL}$ geneticin, $0.15 \mathrm{mg} / \mathrm{mL}$ Zeocin ${ }^{\mathrm{TM}}$ (Invitrogen), and $10 \%$ bovine calf serum. The cells were grown at $37^{\circ} \mathrm{C}$ in an atmosphere of $5 \%$ $\mathrm{CO}_{2}$ in air and $100 \%$ relative humidity. This mouse embryonal NIH $3 \mathrm{~T} 3$ cell line contains a stably transfected luciferase reporter with eight copies of the consensus Gli binding site. ${ }^{25}$ Cyclopamine samples were dissolved in ethanol and added to DMEM media containing 0.5\% bovine calf serum.

Reporter Assay: Shh-Light II cells were seeded in a 96-well plate and grown to complete confluence in the media described above. When cells were confluent, the media was replaced with DMEM supplemented with $0.5 \%$ bovine calf serum, and treated with $0.1 \mathrm{ng}$ of $\mathrm{N}$-terminal mouse recombinant Shh (R\&D Systems, Minneapolis, Minnesota) dissolved in DMEM, and select cyclopamine treatment. To determine the effect of extraction technique on the biological activity of cyclopamine, the collected material from each of the eight extraction methods and three commercial cyclopamine standards were used to create treatments that resulted in final concentrations of $5,1,0.5,0.1$, or 0 (positive control) $\mu \mathrm{M}$ cyclopamine. In each experiment, the controls and treatment wells contained all vehicles, with a final ethanol concentration of $0.05 \%$. Gli activity in the Shh-Light II cell line was assayed $48 \mathrm{~h}$ after treatment with Shh protein and selected compounds using the Dual-Luciferase Reporter Assay System (Promega, Madison, Wisconsin). The Gli-activity was measured by luminescence emitted from cells using a BioTek Synergy H1m Microplate reader. Each experiment was performed thrice. 


\section{Results and Discussion}

HPLC chromatograms for crude extracts representative of each of the eight extraction methods are shown in Figure 1. When necessary, crude samples were spiked with standard to definitively identify the peak corresponding to cyclopamine. Alkaloids typically found in $V$. californicum are shown in Supplementary S1. Each extraction technique yielded a unique array of compounds with differing concentrations of alkaloids in relation to the amount of cyclopamine. The complex sample matrices influence the elution time of cyclopamine as compared to the isolated standard, so peak verification was necessary for correct identification.

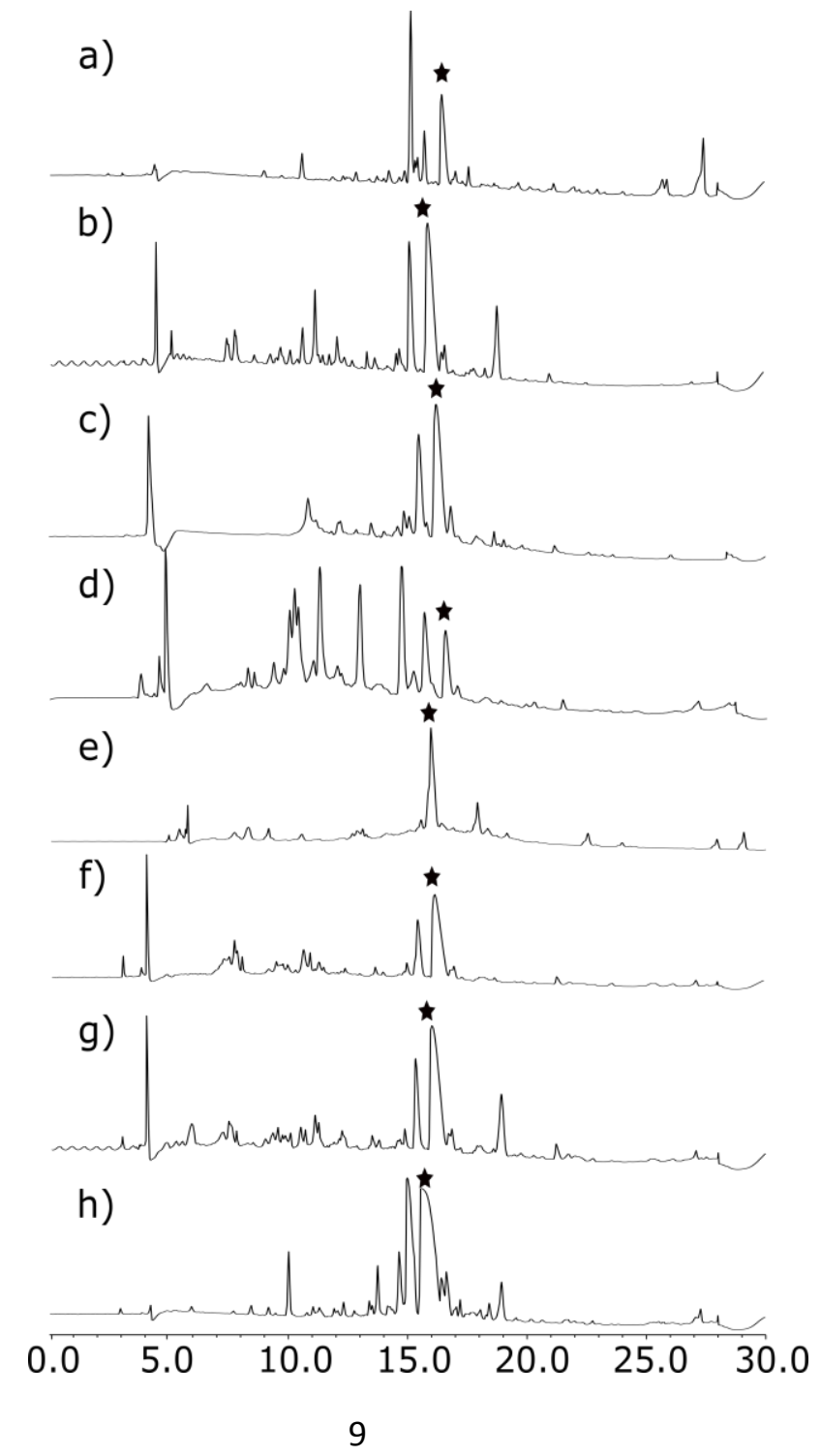


Figure 1. Chromatograms for alkaloid mixtures collected over a 30 minute run time. All chromatograms are shown over the same intensity range (y-axis), and the isolated peak is indicated by asterisks. Extraction conditions are as follows: a) benzene soak, b) ethanol soak, c) microwave assisted, d) supercritical $\mathrm{CO}_{2}$, e) acidic ethanol Soxhlet, f) alkaline ethanol Soxhlet, g) neutral ethanol Soxhlet, and h) benzene Soxhlet.

Soxhlet reflux with ethanol was found to be optimal when the extraction time was allowed to proceed for 6 hours (Supplemental S2). This time was determined by testing the extraction efficiency at six points: 1, 2, 4, 6, 8 and 24 hours. As extraction time was allowed to increase there was a corresponding increase in extracted cyclopamine, which achieved a maximum at 6 hours after which cyclopamine quantity was observed to decrease. It was assumed that prolonged exposure to elevated temperatures degraded cyclopamine, but the nature of the degradation was not investigated.

Cyclopamine from each extraction technique was quantified from crude samples by HPLC-CAD, and a standard curve generated from commercially available cyclopamine (Supplemental S3, $\mathrm{R}^{2}=0.9972$ ). The concentration of cyclopamine in crude alkaloid extracts was determined and the efficiency of the cyclopamine extraction was calculated as a function of dry material from which it was extracted. The cyclopamine extraction results are summarized in Table 1. 


\begin{tabular}{|l|c|c|c|c|}
\hline Extraction Method & $\begin{array}{c}\text { Average } \\
(\mathbf{m g} / \mathbf{g})\end{array}$ & $\begin{array}{c}\text { Standard Deviation } \\
\text { (mg/g) }\end{array}$ & $\begin{array}{c}\text { Relative \% } \\
\text { Recovery }\end{array}$ & $\begin{array}{c}\text { Relative Gli-Reporter } \\
\text { Activity at } \mathbf{0 . 1} \boldsymbol{\mu M}\end{array}$ \\
\hline Ethanol Soak & 8.03 & 0.13 & 100.0 & $40.45 \pm 8.49$ \\
\hline Benzene Soak & 0.39 & 0.18 & 4.9 & $42.68 \pm 2.74$ \\
\hline Benzene Sox & 0.95 & 0.32 & 11.8 & $45.17 \pm 7.63$ \\
\hline Ethanol Sox & 0.75 & 0.14 & 9.3 & $57.10 \pm 7.29$ \\
\hline Ethanol Sox (acidic) & 0.11 & 0.02 & 1.4 & $\mathbf{9 1 . 1 0 \pm 5 . 9 8 *}$ \\
\hline Ethanol Sox (Basic) & 0.73 & 0.11 & 9.1 & $40.14 \pm 8.61$ \\
\hline Supercritical & 0.66 & 0.1 & 8.2 & $\mathbf{9 0 . 4 0 \pm 6 . 5 0 *}$ \\
\hline Microwave & 1.19 & 0.53 & 14.8 & $65.16 \pm 8.63$ \\
\hline
\end{tabular}

Table 1. Comparison of extraction efficiency and corresponding bioactivity of cyclopamine.

Table 1 shows the mg of cyclopamine extracted per $\mathrm{g}$ of initial biomass. Each extraction was repeated in triplicate, and the standard deviation of the yield is shown. The percent recovery relative to ethanol soak extraction is shown for comparison between extraction methods, because recovery from ethanol soak was superior to all other methods. The activity of cyclopamine isolated by each extraction technique to inhibit Hh signaling at $0.1 \mu \mathrm{M}$ is also shown, where comparable high inhibition was observed for ethanol and benzene soak, and Soxhlet under conditions of benzene solvent or alkaline ethanol, and poor bioactivity was measured for acidic ethanol Soxhlet and supercritical fluid extraction methods. In Table 1, the acidic ethanol Soxhlet and supercritical extracts are indicated in bold font and by an asterisks to show reduced biological activity compared to the cyclopamine standard, which measured at $58.08 \pm 7.51$ percent reduction in Gli-reporter activity at $0.1 \mu \mathrm{M}$ cyclopamine treatment.

Recovered cyclopamine by ethanol soak was substantially greater than other techniques, yielding $8.03 \mathrm{mg} / \mathrm{g}$, compared to 0.39 to $1.19 \mathrm{mg} / \mathrm{g}$. Based on literature precedent, it is likely that prolonged ethanol exposure results in deglycosylation of cycloposine to cyclopamine, while 
simultaneously providing a mild solvent condition that prevents cyclopamine degradation. ${ }^{20}$ The acidic ethanol Soxhlet extraction yielded virtually no detectable cyclopamine of the correct $\mathrm{m} / \mathrm{z}$. Despite this, as indicated in Figure 1, the major peak in the acidic ethanol Soxhlet extraction was collected and evaluated for inhibitory activity towards Hh signaling.

All extraction methods yielded a white, amorphous powder upon isolation except the acidic ethanol Soxhlet and supercritical extraction methods. These methods yielded brown and $\tan$ powders, respectively. Cyclopamine has been reported to degrade to veratramine ${ }^{23}$ or isomerize under acidic conditions, resulting in reduced bioactivity. ${ }^{24}$ Therefore, it was anticipated that extraction by acidic ethanol Soxhlet reflux would yield a product of diminished effectiveness at inhibiting Hh signaling. High resolution QTOF MS showed the product obtained under acidic conditions was characterized by an $\mathrm{m} / \mathrm{z}$ and fragmentation pattern consistent with veratramine, ${ }^{22}$ with a $[\mathrm{M}+\mathrm{H}]^{+}$peak of 410.304 , and major fragmentation peaks of 295.2, 114.1, 183.1, 211.1, 171.1, and 84.1 (Figure 2). 


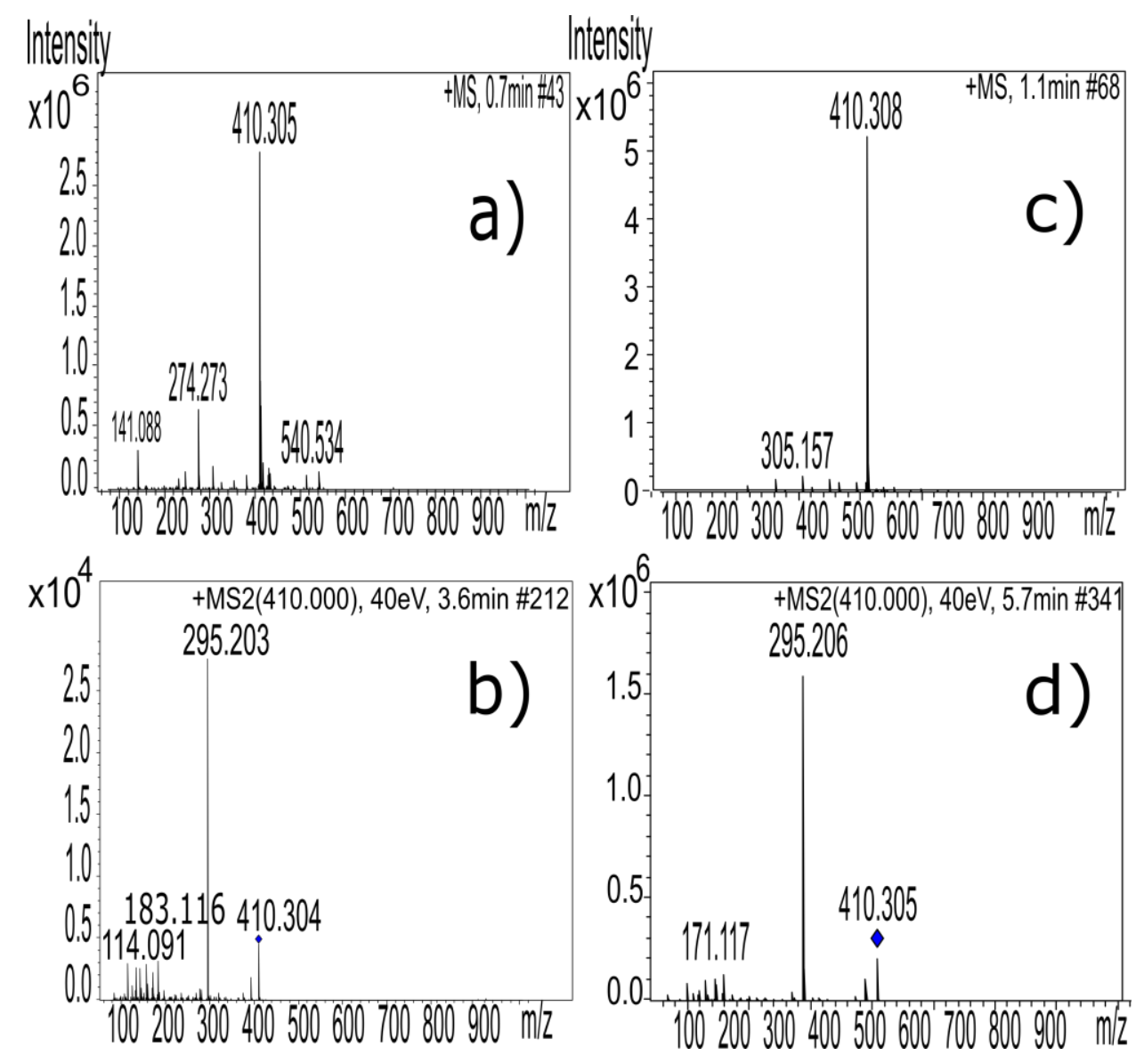

Figure 2. Direct comparison of MS and MS/MS of the acidic ethanol Soxhlet isolated product and veratramine standard. a) High resolution MS analysis of acidic ethanol Soxhlet extracted product, and b) MS/MS analysis of isolated ion of $\mathrm{m} / \mathrm{z}$ of 410.0. c) High resolution MS analysis of veratramine standard, and d) MS/MS analysis of isolated ion of m/z of 410.0.

To confirm that each extraction method yielded biologically active cyclopamine capable of Hh pathway inhibition, the isolated cyclopamine was tested alongside three commercially available cyclopamine standards from different suppliers in an Hh-responsive fibroblast cell line Shh-Light II assay. ${ }^{25}$ Extracted cyclopamine from all methods was able to decrease Shh peptidestimulated Gli reporter activity at a level equivalent to the cyclopamine standards for each concentration tested, except for supercritical $\mathrm{CO}_{2}$ and acidic ethanol Soxhlet (Figure 3). 

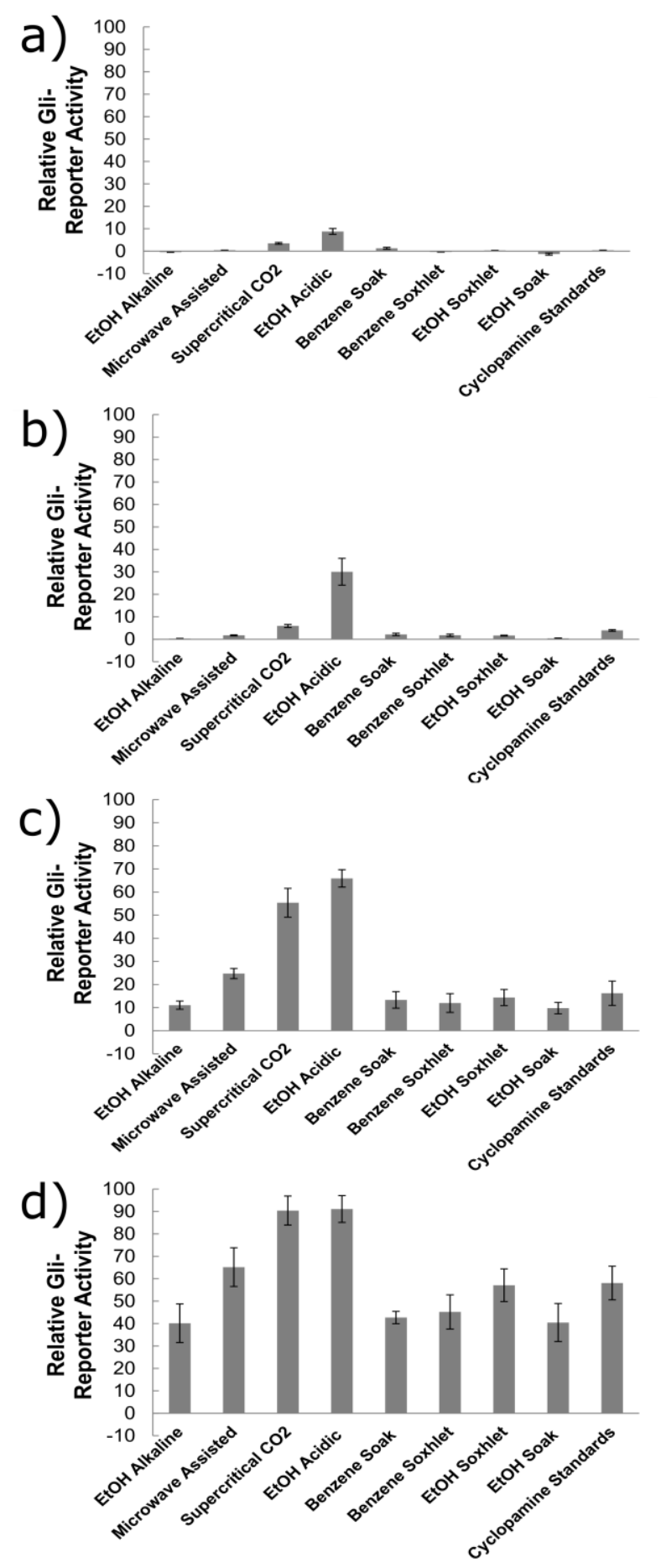

Figure 3. Effects of extract on Shh-stimulated Gli-responsive promoter in the Shh-Light II cell line. Shh-Light II cells were treated with various compounds in the presence of $2 \mu \mathrm{g} / \mathrm{mL}$ mouse recombinant Shh for $48 \mathrm{~h} . \mathrm{a}), \mathrm{b}), \mathrm{c}$ ), and d) correspond to 5, 1, 0.5, and $0.1 \mu \mathrm{M}$ cyclopamine, 
respectively, for each extraction method. Experiments were performed in triplicate with standard deviation indicated.

Included in Figure 3 is average of the triplicate trials of the three commercial standards and a side-by-side comparison of each standard is shown in Supplemental S4. With the exception of the supercritical $\mathrm{CO}_{2}$ and acidic ethanol Soxhlet extraction methods, each technique and the standard control resulted in nearly complete $(>98 \%)$ inactivation of the Shh peptide-stimulated Hh pathway activity at both $5 \mu \mathrm{M}$ and $1 \mu \mathrm{M}$, and an average of $13.5 \pm 5.3 \%$ and $49.4 \pm 9.8 \%$ of reported activity at $0.5 \mu \mathrm{M}$ and $0.1 \mu \mathrm{M}$, respectively. Based upon the similarities observed in the biological activity, all extraction techniques except the supercritical fluid and acidic ethanol Soxhlet can be presumed to have yielded cyclopamine with chemical and isomeric purity equivalent to that of the three commercial cyclopamine standards tested. The purity of the standards was confirmed to be $>98 \%$ by integrating the peak area of the high resolution QTOF MS (see Supplemental S5).

Inhibition of Hh signaling was observed in Shh-Light II treated with acidic ethanol Soxhlet isolated product, although at considerably decreased potency compared to other extraction techniques and cyclopamine standards. Gli-reporter activity was measured at $8.8 \pm 1.3 \%, 30.0 \pm 6.0 \%, 65.9 \pm 3.8 \%$, and $91.1 \pm 6.0 \%$ for $5,1,0.5$, and $0.1 \mu \mathrm{M}$ samples, respectively. To further investigate the identity of this acid degraded product, the inhibitory activity of veratramine standard on Hh signaling in Shh-Light II cells was performed. Inhibition was observed in the cells treated with the standard, but it was markedly less potent than the acid degraded product. The relative Gli-report activity measured at $72.0 \%$ for the cells treated with 1 $\mu \mathrm{M}$ veratramine standard, compared to $30.0 \%$ activity for cells treated with $1 \mu \mathrm{M}$ acid degraded product (see Supplemental S6). 
This may be due to presence of additional alkaloids in acidic ethanol extract, such as peaks observed at $\mathrm{m} / \mathrm{z} 540.534,416.351$ and 430.330 as shown in the MS (Figure 2a), and trace quantities of residual cyclopamine. Veratramine has been reported to be ineffective in blocking hedgehog signaling in chick embryos at $240 \mathrm{nM},{ }^{7}$ but acid treated cyclopamine has been shown to inhibit Hh signaling with a diminished potency in Shh-Light II cells at $1 \mu \mathrm{M} .{ }^{18}$ These discrepancies could be the result of the disparate techniques and concentrations used to evaluate disruption of Hh signaling.

Decreased bioactivity for cyclopamine extracted by supercritical fluid was also observed. The high resolution QTOF MS of this sample showed a veratramine contaminate, as evidenced by a $[\mathrm{M}+\mathrm{H}]^{+}$of 410.3 . Veratramine in the supercritical extract was observed in each of the triplicate trials, indicating the crude sample matrix from this extraction technique made the separation and isolation of pure cyclopamine less efficient on the semi-preparative column. In the acidic ethanol Soxhlet extractions spiked with cyclopamine standard, there are clearly distinct peaks observed for veratramine and cyclopamine (Supplemental S7). This is not the case for the supercritical fluid extraction, indicating that the unique constituents of the crude supercritical fluid extract made collection of cyclopamine independent of veratramine more difficult. However, it is not immediately clear why this phenomenon is observed. From integration of MS peaks, the supercritical extract yielded cyclopamine that was 79.4\% pure, with the remainder of the mixture being primarily veratramine (Supplemental S5c and S5d).

\section{Conclusion}

Most of the extraction techniques evaluated in this study yielded cyclopamine with purity that is comparable to commercially available sources, as demonstrated in Hh signaling inhibition assay. The ethanol soak method yielded substantially higher quantities of cyclopamine compared 
to the other methods. Acidic ethanol Soxhlet extraction causes the cyclopamine to degrade. This degradation product is most likely veratramine, although it demonstrated Hh inhibition at considerably increased potency compared to commercially available veratramine standard. Veratramine was also present in the supercritical fluid extract because the semi-preparative HPLC column could not completely separate these closely related chemical species. The identity of the unique assortment of alkaloids resulting from each extraction technique warrants further investigation, as does the precise chemical nature of the acid degraded product.

\section{Acknowledgements}

We would like to acknowledge the contributions of Erik Sheldon, Emily Drussel, and Chris Chandler of Boise State University, and Edna Rey-Vizgirdas of the Boise National Forest. We thank Barb Jibben of the Biomolecular Research Center at Boise State University for the constructive comments and critical review of this publication. The project described was supported by Institutional Development Awards (IDeA) from the National Institute of General Medical Sciences of the National Institutes of Health under Grants \#P20GM103408 and P20GM109095. We also acknowledge support from The Biomolecular Research Center at Boise State with funding from the National Science Foundation, Grants \# 0619793 and \#0923535; the MJ Murdock Charitable Trust; and the Idaho State Board of Education. Its contents are solely the responsibility of the authors and do not necessarily represent the official views of NIH. 


\section{References and Notes}

1. McNeal, D. W.; Shaw, A. D. Veratrum. In: Flora of North America Editorial Committee, eds. 2002. Flora of North America North of Mexico. New York and Oxford. Vol. 26, pp/ 72-76.

2. Chandler, C. M.; McDougal, O. M. Phytochem. Rev. 2014, 13(3) 671-694.

3. Keeler, R. F.; Binns, W. Teratology 1968, 1, 5-10.

4. Keeler, R. F. Proc. Soc. Exp. Biol. Med. 1973, 142, 1287-1291.

5. Keeler, R. F. Lipids 1978, 13, 708-715.

6. Cooper, M. K.; Porter, J. A.; Young, K. E.; Beachy, P.A. Science 1998, 280, 1603-1607.

7. Incardona, J. P.; Gaffield, W.; Kapur, R. P.; Roelink, H. Development 1998, 125, 35533562.

8. Heretsch, P.; Tzagkaroulaki, L.; Giannis, A. Angew. Chem. Int. Ed. 2010, 49, 3418-3427.

9. Onishi, H.; Katano, M. Cancer Sci. 2011, 102, 1756-1760.

10. Ok, C. Y.; Singh, R. R.; Vega, F. Am. J. Pathology 2012, 180, 2-11.

11. Wilkinson, S. E.; Furic, L.; Buchanan, G.; Larsson, O.; Pedersen, J.; Frydenberg, M.; Risbridger, G. P.; Taylor, R. A. The Prostate 2013, 73, 1810-1823.

12. Matsushita, S.; Onishi, H.; Nakano, K.; Nagamatsu, I.; Imaizumi, A.; Hattori, M.; Oda, Y.; Tanaka, M.; Katano, M. Cancer Sci. 2014, 105, 272-280.

13. Onishi, H.; Kai, M.; Odate, S.; Iwasaki, H.; Morifuji, Y.; Ogino, T.; Morisaki, T.; Nakashima, Y.; Katano, M. Cancer Sci. 2011, 102, 1144-1150.

14. Wong, C. S.; Strange, R. C.; Lear, J. T.; Basal Cell Carcinoma, BMJ 2003, 327, 794-798.

15. Tremblay, M. R.; Lescarbeau, A.; Grogan, M. J.; Tan, E.; Lin, G.; Austad, B. C.; Yu, L.; Behnke, M. L.; Nair, S. J.; Hagel, M.; White, K.; Conley, J.; Manna, J. D.; Alvarez-Diez, T. 
M.; Hoyt, J.; Woodward, C. N.; Sydor, J. R.; Pink, M.; MacDougall, J.; Campbell, M. J.;

Cushing, J.; Ferguson, J.; Curtis, M. S.; McGovern, K.; Read, M. A.; Palombella, V. J.;

Adams, J.; Castro, A. C. J. Med Chem. 2009, 52, 4400-4418.

16. ClinicalTrials.gov, Pilot study of cetuximab and the hedgehog inhibitor IPI-926 in recurrent head and neck cancer. http://clinicaltrials.gov/show/NCT01255800, 2013 (accessed 1.11.16).

17. National Cancer Institute, FDA Approval for Vismodegib. http://www.cancer.gov/cancertopics/druginfo/fda-vismodegib/, 2012 (accessed 5.5.2013).

18. Keeler, R. F.; Binns, W. Can. J. Biochem. 1966, 44, 819-828.

19. Oatis, J. E.; Brunsfeld, P.; Rushing, J. W.; Moeller, P. D.; Bearden, D. W.; Gallien, T. N.; Cooper, G. Chem. Cent. J. 2008, 2:12, 1-6.

20. Splinter, S.; Kadali, S. U.S. Patent Application 20110160457, 2011.

21. Jayatilake, G. S.; Richheimer, S. L. Mann, D.A.; U.S. Patent Application 20100003728, 2010.

22. Chandler, C. M.; Habig, J. W.; Fisher, A. A.; Ambrose, K. V.; Jiménez, S. T.; McDougal, O. M.; Nat. Prod. Commun. 2013, 8.

23. Keeler, R. F.; Teratology 1970, 3 175-180.

24. Wilson, S. R.; Strand, M. F.; Krapp, A.; Rise, F.; Petersen, D.; Krauss, S.; J. Pharm. Biomd. Anal. 2010, 52 707-713.

25. Sasaki, H.; Hui, C.; Nakafuku, M.; Kondoh, H.; Development 1997, 124 1313-22. 